\title{
Purine-Nucleoside Phosphorylase Deficiency
}

National Cancer Institute

\section{Source}

National Cancer Institute. Purine-Nucleoside Phosphorylase Deficiency. NCI Thesaurus.

Code C3963.

PNP reversibly catalyzes the phosphorolysis of the purine nucleosides, (deoxy)inosine and (deoxy)guanosine, to their respective purine bases and the corresponding ribose-1phosphate. Deficiency in this enzyme is an autosomal recessive cause of combined immunodeficiency. 